\title{
Turbulent resistivity evaluation in magnetorotational instability generated turbulence
}

\author{
G. Lesur ${ }^{1}$ and P.-Y. Longaretti ${ }^{2}$ \\ 1 Department of Applied Mathematics and Theoretical Physics, University of Cambridge, Centre for Mathematical Sciences, \\ Wilberforce Road, Cambridge CB3 0WA, UK \\ e-mail: g.lesur@damtp.cam.ac.uk \\ ${ }^{2}$ Laboratoire d'Astrophysique, UJF CNRS, BP 53, 38041 Grenoble Cedex 9, France
}

Received 3 April 2009 / Accepted 26 June 2009

\begin{abstract}
Context. MRI turbulence is a leading mechanism for the generation of an efficient turbulent transport of angular momentum in an accretion disk through a turbulent viscosity effect. It is believed that the same process could also transport large-scale magnetic fields in disks, reshaping the magnetic structures in these objects. This process, known as turbulent resistivity, has been suggested and used in several accretion-ejection models and simulations to produce jets. Still, the efficiency of MRI-driven turbulence to transport largescale magnetic fields is largely unknown.

Aims. We present new analytical and numerical results aiming at quantifying the turbulent resistivity produced by MRI-driven turbulence in accretion disks.

Methods. We investigate this problem both analytically and numerically. We introduce a linear calculation of the MRI in the presence of a spatially inhomogeneous mean magnetic field. We show that, in this configuration, MRI modes lead to an efficient magnetic field transport, on the order of the angular momentum transport. We next use fully non linear simulations of MRI turbulence to compute the turbulent resistivity in several magnetic configurations.

Results. We find that the turbulent resistivity is on the order of the turbulent viscosity in all our simulations, although somewhat lower. The variations in the turbulent resistivity are correlated with the variation in the turbulent viscosity as a function of the imposed mean field. Finally, the turbulent resistivity tensor is found to be highly anisotropic with a diffusion coefficient 3 times greater in the radial direction than in the vertical direction.

Conclusions. These results support the possibility of driving jets from turbulent disks; the resulting jets may not be steady.
\end{abstract}

Key words. accretion, accretion disks - turbulence - magnetohydrodynamics (MHD)

\section{Introduction}

After several decades of debate, and in spite of significant progress, the origin and efficiency of angular momentum transport remains a central problem in accretion disks physics. Turbulence is one of the major physical mechanism through which the observationally well-constrained anomalous transport can be achieved; indeed, the first $\alpha$ model (Shakura \& Sunyaev 1973) already assumed a strong level of turbulence, leading to an effective viscosity orders of magnitude higher than molecular viscosity. However, the physical origin of this turbulence in disks is still largely discussed.

Various sources of turbulence, both hydrodynamical and magneto-hydrodynamical, have been proposed. Subcritical turbulence (Richard \& Zahn 1999, and references therein), if present, is too inefficient (Lesur \& Longaretti 2005; Ji et al. 2006). Convection is also too inefficient and transports angular momentum in the wrong direction (Cabot 1996; Stone \& Balbus 1996). Two-dimensional turbulence driving by small-scale, incoherent gravitational instabilities (Gammie 1996) or baroclinic instabilities (Klahr \& Bodenheimer 2003) is a possible option, although the last one is still highly controversial (Johnson \& Gammie 2006; Petersen et al. 2007).

In a seminal paper, Balbus \& Hawley (1991) indentify an MHD instability, the magnetorotational instability (MRI) that drives turbulence in the nonlinear regime. This instability provides the most extensively studied transport mechanism, mainly with the help of local unstratified (Hawley et al. 1995) and stratified (Stone et al. 1996) 3D simulations, and global (Hawley 2000) disk simulations. These simulations have shown that MRI turbulence is an efficient way to transport angular momentum, although the role of microphysical processes has largely been underestimated (Lesur \& Longaretti 2007; Fromang et al. 2007).

MRI turbulence may also produce resistive transport ${ }^{1}$ (transport of magnetic fields through a "turbulent resistivity" process) in disks. This transport is a key ingredient of accretion-ejection models (see, e.g., Ferreira 1997; Casse \& Ferreira 2000, and references therein). The related turbulent resistivity $\eta_{\mathrm{T}}$ is parameterized with the Shakura-Sunyaev ansatz as $\eta_{\mathrm{T}}=\alpha_{\eta} V_{\mathrm{A}} H\left(V_{\mathrm{A}}\right.$ is the Alfvén speed based on the mean field); stationary accretion ejection models require an anisotropy of the turbulent resistivity transport of few; furthermore, a very efficient turbulent transport with $\alpha_{\eta} \lesssim 1$ is required to produce stationary structures. Less efficient transport would not prevent the launching of jets from disks, but these structures would then be nonstationary. The question of whether turbulent resistivity is an adequate source of field line slippage through the gas to launch a jet is

\footnotetext{
${ }^{1}$ In this paper, a transport process refers to any physical mechanism by which a magnetic field line is moved from one place to another, without assuming anything about flux-freezing conditions.
} 
still controversial in the literature, and is further discussed in the concluding section of this paper.

In any case, turbulent resistivity is an issue in its own right, and in this paper, we present new numerical results aimed at quantifying more precisely the resistive transport due to MRI turbulence. Our approach differs from the method recently introduced by Brandenburg, Rädler, Schrinner and coworkers to compute the turbulent resistivity tensor (see Schrinner et al. 2005; Brandenburg et al. 2008, and references therein). Their method relies on an expansion of the mean electromotive force with respect to the mean field and its derivatives, and makes use of appropriately chosen test field, whose evolution is computed along with the turbulent flow. Our approach is also different from the one used by Guan \& Gammie (2009), in which a magnetic structure is imposed as an initial condition, and the resistive properties of the background turbulent flow are deduced from the decay time of this structure. The method we adopt here is described in Sect. 2, and the physical content of the two methods is discussed there. More generally, Sect. 2 describes the physics and the numerical methods we have used to study turbulent resistivity in disks. Section 3 presents a linear analysis of the model, which sheds some light on the numerical findings described in Sect. 4. Section 5 discusses the astrophysical implications of these results along with some future line of work.

\section{Shearing-box equations and numerical method}

MRI-related turbulence has been extensively studied in the literature, but little attention has been devoted to the question of turbulent-driven resistivity yet. One of the major advances of the recent years has been the realization that an accurate determination of turbulent transport properties requires an accurate representation of all scales down to the dissipation scales (Lesur \& Longaretti 2007; Fromang et al. 2007), although this accuracy has not yet been achieved for astrophysically relevant magnetic Prandtl and Reynolds numbers regimes. We do not imply that actual astrophysical Reynolds numbers need to be resolved in simulations to obtain reliable results, an obviously hopeless task anyway; but for large enough Reynolds and magnetic Reynolds numbers, one expects the dissipation scale to be decoupled from the transport scales, in which case one could use a closure model without resolving the dissipation scales. However, today simulations don't seem to have reached this regime yet.

Shearing box simulations offer a particularly convenient setting to quantify turbulent resistivity; however, the boundary conditions prevent the existence of the large scale gradients of mean magnetic field required for characterizing the transport of this mean field produced by turbulence. We bypass this difficulty by a prescription whose physical motivation and formulation will be described later on in this section. For the time being, we briefly recall the basic equations for the shearing-box model, which has been largely studied and used in the literature. The reader may consult Hawley et al. (1995), Balbus (2003) and Regev \& Umurhan (2008) for an extensive discussion of the properties and limitations of this model.

Since MHD turbulence in disks is essentially subsonic, we will work in the incompressible approximation, which allows us to eliminate sound waves and density waves from the problem. Although density waves are excited in shearing box turbulence (Heinemann \& Papaloizou 2008b) they should not have a big impact on the turbulence spectrum itself or on turbulent transport as their amplitude decays exponentially fast when one goes to small scales (Heinemann \& Papaloizou 2008a). This has also been confirmed by direct comparison between incompressible and compressible simulations (Fromang et al. 2007). We also neglect vertical stratification, consistently with the local shearingbox model (Regev \& Umurhan 2008). Explicit molecular viscosity and resistivity are included in our description.

The shearing box equations follow from a local approximation. We chose a Cartesian box centered at $r=R_{0}$, rotating with the disk at angular velocity $\Omega=\Omega\left(R_{0}\right)$ and having dimensions $\left(L_{x}, L_{y}, L_{z}\right)$ with $L_{i} \ll R_{0}$. Assuming $R_{0} \phi \rightarrow x$ and $r-R_{0} \rightarrow-y$, one eventually obtains the shearing box equations:

$$
\begin{aligned}
\partial_{t} \boldsymbol{U}+\boldsymbol{\nabla} \cdot(\boldsymbol{U} \otimes \boldsymbol{U})= & -\boldsymbol{\nabla} \Pi+\boldsymbol{\nabla} \cdot(\boldsymbol{B} \otimes \boldsymbol{B}) \\
& -2 \boldsymbol{\Omega} \times \boldsymbol{U}+2 \Omega S y \boldsymbol{e}_{\boldsymbol{y}}+v \boldsymbol{\Delta} \boldsymbol{U}, \\
\partial_{t} \boldsymbol{B}= & \boldsymbol{\nabla} \times(\boldsymbol{U} \times \boldsymbol{B})+\eta \boldsymbol{\Delta} \boldsymbol{B}, \\
\boldsymbol{\nabla} \cdot \boldsymbol{U}= & 0 \\
\boldsymbol{\nabla} \cdot \boldsymbol{B}= & 0 .
\end{aligned}
$$

The boundary conditions associated with this system are periodic in the $x$ and $z$ direction and shearing-periodic in the $y$ direction (Hawley et al. 1995). In these equations, we have defined the mean shear $S=-r \partial_{r} \Omega$, which is set to $S=(3 / 2) \Omega$, assuming a Keplerian rotation profile. The generalized pressure term $\Pi$ includes both the gas pressure term $P / \rho_{0}$ and the magnetic one $\boldsymbol{B}^{2} / 2 \rho_{0}$. This generalized pressure $\Pi$ is actually a Lagrange multiplier enforcing Eq. (3), and is therefore computed by solving a Poisson equation. Note also that the magnetic field is expressed in Alfvén-speed units, for simplicity.

The steady-state solution to these equations is the local Keplerian profile $\boldsymbol{U}=S \boldsymbol{y} \boldsymbol{e}_{\boldsymbol{x}}$. In this paper, we will consider only the turbulent deviations from this Keplerian profile. These may be written as $\boldsymbol{V}=\boldsymbol{U}-\boldsymbol{S} y \boldsymbol{e}_{\boldsymbol{x}}$, leading to the following equations for $\boldsymbol{V}$ :

$$
\begin{aligned}
\partial_{t} \boldsymbol{V}+\boldsymbol{\nabla} \cdot(\boldsymbol{V} \otimes \boldsymbol{V})= & -\boldsymbol{\nabla} \Pi+\boldsymbol{\nabla} \cdot(\boldsymbol{B} \otimes \boldsymbol{B})-S y \partial_{x} \boldsymbol{V} \\
& +(2 \Omega-S) V_{y} \boldsymbol{e}_{x}-2 \Omega V_{x} \boldsymbol{e}_{y}+v \boldsymbol{\Delta} \boldsymbol{V}, \\
\partial_{t} \boldsymbol{B}= & -S y \partial_{x} \boldsymbol{B}+S B_{y} \boldsymbol{e}_{x} \\
& +\boldsymbol{\nabla} \times(\boldsymbol{V} \times \boldsymbol{B})+\eta \boldsymbol{\Delta} \boldsymbol{B} \\
\boldsymbol{\nabla} \cdot \boldsymbol{V}= & 0 \\
\boldsymbol{\nabla} \cdot \boldsymbol{B}= & 0
\end{aligned}
$$

Following Hawley et al. (1995), one can integrate the induction Eq. (6) over the volume of the box, leading to:

$$
\frac{\partial\langle\boldsymbol{B}\rangle}{\partial t}=S\left\langle B_{y}\right\rangle \boldsymbol{e}_{x}
$$

where \langle\rangle denotes a volume average. Therefore, the mean magnetic field is conserved, provided that no mean radial field is present. In this work, the mean field will be either vertical or azimuthal.

To numerically solve the shearing-box equations, we use a spectral Galerkin representation of Eqs. (5)-(8) in the sheared frame (see Lesur \& Longaretti 2005). This frame allows us to use a Fourier decomposition since the shearing-sheet boundary conditions are transformed into perfectly periodic boundary conditions. Moreover, this decomposition allows us to conserve magnetic flux to machine precision without any modification, which is an advantage compared to finite-difference or finitevolume methods (the total magnetic flux created during one simulation is typically $10^{-11}$ ). Equations (7) and (8) are enforced to machine precision using a spectral projection (Peyret 2002). The nonlinear terms are computed with a pseudospectral method, and aliasing is prevented using the $3 / 2$ rule. To always compute the physically relevant scales in the sheared frame, we use a remap method similar to the one described by 
Umurhan \& Regev (2004). This routine redefines the sheared frame every $T_{\text {remap }}=L_{x} /\left(L_{y} S\right)$ and we have checked that none of the behaviour we describe in this paper was related to this numerical timescale. Since spectral methods are very little dissipative by nature, we check that numerical dissipation is kept to very small values, computing the total energy budget at each time step (see Lesur \& Longaretti 2005, for a discussion of this procedure). We therefore ensure that numerical dissipation is responsible for less than $3 \%$ of the total dissipative losses occurring in these simulations.

To quantify the dissipation processes in the simulations, we use dimensionless numbers defined as:

- the Reynolds number, $R e=S L_{z}^{2} / v$, comparing the nonlinear advection term to the viscous dissipation;

- the magnetic Reynolds number, $R m=S L_{z}^{2} / \eta$, comparing magnetic field advection to the Ohmic resistivity;

- the magnetic Prandtl number, defined as the ratio of the two previous quantities $P m=R m / R e=v / \eta$, which measures the relative importance of the dissipation processes, and, correlatively, is related to the ratio of the viscous and resistive dissipation scales.

In the following we use $S^{-1}$ as the unit of time and $S L_{z}$ as the unit of velocity. One orbit corresponds to $T_{\text {orb }}=3 \pi S^{-1}$. For simplicity, we keep the same notation for dimensionless and dimensional quantities.

\subsection{Turbulent resistivity definition}

Our aim is to test to which extent the effect of MRI turbulence on the mean field can be modelled as a turbulent resistivity on large scales, and characterize inasmuch as possible the resulting turbulent resistivity tensor. We therefore distinguish the large scale mean field $\overline{\boldsymbol{B}}$ and velocity $\overline{\boldsymbol{V}}$ and the fluctuating (turbulent) fields $\boldsymbol{b}$ and $\boldsymbol{v}$. We assume $\langle\boldsymbol{b}\rangle=0$ and $\langle\boldsymbol{v}\rangle=0$ where \langle\rangle denotes an ensemble (or time, under the ergodic hypothesis) average. The induction equation reads:

$\partial_{t} \overline{\boldsymbol{B}}=\nabla \times(\overline{\boldsymbol{V}} \times \overline{\boldsymbol{B}})+\nabla \times \mathcal{E}$

where we have defined the mean electromotive force $($ EMF) $\mathcal{E}=$ $\langle\boldsymbol{v} \times \boldsymbol{b}\rangle$. The turbulent resistivity hypothesis assumes:

$\mathcal{E}_{i}=\beta_{i j k} \partial_{x_{k}} \bar{B}_{j}$

Our main objective is to test and quantify this assumption. We keep no term proportional to $\bar{B}$ ( $\alpha$-like effect), as we found out that they are not produced in our simulations.

At this point, two different routes are open to study the turbulent diffusion of the magnetic field.

- In the first one, Eq. (11) is assumed. One can split Eq. (6) into an equation for the mean field $\bar{B}$ and one for the deviation from the mean $\boldsymbol{b}$. As the induction equation is linear in the field, $\boldsymbol{b}$ formally depends on the mean and turbulent velocity fields and mean magnetic field only. One then introduces extraneous magnetic fields $\boldsymbol{b}^{p q}$, whose equation of evolution is the same as for $\boldsymbol{b}$, except for the mean field $\bar{B}$ which is replaced by a ad hoc test field $\bar{B}^{p q}$. It is then possible to derive the EMF associated to this test field $\mathcal{E}^{p q}=\left\langle\boldsymbol{v} \times \boldsymbol{b}^{p \boldsymbol{q}}\right\rangle$.

One can deduce some of the components of $\beta_{i j k}$ using several test fields and computing the correlations between $\overline{B^{p q}}$ and $\mathcal{E}^{p q}$. By construction, this method probes the velocity field $v$ with a test field $\boldsymbol{B}^{p q}$ which is different from the real field $\boldsymbol{B}$ entering in the Lorentz force. Therefore, one assumes implicitly that the properties of $\beta_{i j k}$ don't depend on the topological properties of $\boldsymbol{B}$, which might not be true for subcritical dynamos (see e.g. Lesur \& Ogilvie 2008) or turbulence driven through the Lorentz force like MRI turbulence. This method is detailed in Schrinner et al. (2005) and Brandenburg et al. (2008) and references therein.

- Alternatively, one can impose the constancy of some large scale component of the magnetic field gradient throughout the evolution and test if Eq. (11) accurately represents the effect of turbulence on field diffusion. As in the previous approach, adequate choices of the imposed field configuration allow us to characterize various elements of the diffusivity tensor. This approach is adopted here and specified in more detail in the following subsection.

These two approaches do not quite probe the same physical aspects of the problem. This first one is justified inasmuch as the mean field hypothesis Eq. (11) holds, provided that the turbulent resistivity tensor does no depend on the field topology. The second one is justified without assumption if the turbulent diffusion of the field is much slower than the other time-scales associated to the turbulence dynamics. However, even if this requirement is not satisfied, the physical content of this second approach can be grasped by comparing the questions of magnetic field and momentum transfer. Consider for example a simple unmagnetized plane Couette flow. With shearing box boundary conditions, the turbulent flow is unable to react of the shear (velocity gradient) imposed on the flow through the boundary conditions. As shown by Lesur \& Longaretti (2005), the flow nevertheless becomes turbulent above some transition Reynolds number $R_{\mathrm{c}}$ and produces a shearwise transport characterized by $R_{\mathrm{c}}$. Alternatively, one can let the flow react on the imposed velocity gradient by adopting e.g. wall boundary conditions in the shearwise direction. This changes has little effect on the transition Reynolds numbers (at least for cyclonic rotation: see Lesur \& Longaretti 2005), and does not appear to affect much the dimensionless transport, as suggested by the numerical results of Lesur \& Longaretti (2005) and the experimental results compiled in Dubrulle et al. (2005). These considerations make it plausible that the turbulent diffusion of the magnetic field with an imposed field gradient or with a self-consistently evolving one should be similar, although a more systematic study is required to ascertain this statement.

In practice, we chose field configurations such that only one component of the field gradient is non vanishing at any given time, so that the current is directly proportional to this nonvanishing gradient. In this situation, one can dispense with the third order tensor and use

$\mathcal{E}_{i}=-\eta_{i j} J_{j}$

instead, where $\boldsymbol{J}=\boldsymbol{\nabla} \times \overline{\boldsymbol{B}}$ is the mean electric current and $\eta$ is a constant tensor, but the reader should keep in mind that there is no reason that $\eta_{i j k}$ should be antisymmetric in the relevant indices. Similarly, $\eta$ has no reason to be diagonal for MRI turbulence. In this work, we explore this model looking for linear correlations between the components $\mathcal{E}_{j}$ and $J_{i}$, and computing the related resistivity tensor coefficients.

To keep in line with the logic of the $\alpha$ parameterization as adapted to an incompressible fluid (see Lesur \& Longaretti 2007), one may introduce an $\alpha$ tensor relating to the turbulent $\eta$ tensor:

$\alpha_{\eta_{i j}} \simeq \frac{\eta_{i j}}{S L_{z}^{2}}$ 
With our choice of units $\left(S=1\right.$ and $\left.L_{z}=1\right)$, one has $\alpha_{\eta_{i j}}=\eta_{i j}$, and the distinction between the two quantities is usually dropped in the remainder of the paper.

\subsection{Numerical protocol}

To compute the turbulent resistivity, one needs a mean current, which is not present in local (shearing box) simulations. We obtain this current by imposing a large scale and non homogeneous field in the box. This is done with the help of our Galerkin representation by forcing the largest Fourier mode in one direction to a constant value $\Delta B_{0}$. To trigger the MRI, we impose a mean field $B_{0}$ which can be either azimuthal or vertical. The aspect ratio is $L_{x} \times L_{y} \times L_{z}=4 \times 2 \times 1$ where $x=\phi, y=-r$ and $z=z$. The factor 2 in $L_{y}$ allow us to trigger more easily secondary instabilities in the strong mean vertical field cases (see Goodman \& Xu 1994; and Bodo et al. 2008). The resolution used is $128 \times 128 \times 64$, similar in cell size to the one used in Lesur \& Longaretti (2007). The Reynolds number is kept constant at $R e=1600$ as well as the magnetic Prandtl number, which is fixed at unity: $P m=1$. This corresponds to a Elsasser number $\Lambda \equiv V_{\mathrm{A}}^{2} / \eta \Omega=24$ for $B_{0}=0.1$ and $\Lambda=1.5$ for $B_{0}=0.025$. Each simulation is integrated over 500 shear times (to obtain meaningful time averages), and the average are computed from 400 last shear times (to avoid initial conditions artifacts).

To postprocess the results, we first compute the time average of $\boldsymbol{B}$ and $\mathcal{E}_{j}$. We then use a script which extracts the mean current and compute the correlation with the emfs, giving in the end one component of the $\eta$ tensor for one run. Note that using this procedure, we can compute resistivity associated with $B_{z}(y)$ (radially varying vertical field), $B_{x}(y)$ (radially varying azimuthal field) and $B_{x}(z)$ (vertically varying azimuthal field) configurations. Imposing a constant radial field $B_{y}$ would lead to a linear growth of the azimuthal field $B_{x}$, which may not have any physical meaning. We cannot compute turbulent resistivity associated with non axisymmetric structures because of the shearing-sheet boundary conditions.

The runs we have performed do not test all the dependencies of the dimensionless turbulent resistive transport with respect to all the dimensionless parameters of the problem, but only a subset of them, namely:

- the role of the mean field orientation $B_{0}$, and of the orientation the imposed field varying component $\Delta B_{0}$;

- the role of the relative amplitude of the mean and imposed varying field components $\epsilon=\Delta B_{0} / B_{0}$. In the process, only a few components of the turbulent resistivity tensor are probed;

- the role of the relative mean field amplitude, characterized by $^{2} \beta=\left(4 \pi \rho S^{2} H^{2}\right) / B_{0}^{2}=B_{0}^{-2}$ (the last equality follows from our choice of units).

No characterization with respect to the dissipation parameters has been attempted (most notably the magnetic Prandtl number), a problem requiring substantial efforts and that will be addressed elsewhere.

Our local simulation box physical size is on the order of the scale height at most. As such, one would expect to mimic the effect of large scale gradients of the magnetic field by small values of $\epsilon$. However sizeable (but of limited extent) local field

\footnotetext{
${ }^{2}$ Our $\beta$ parameter is akin to the usual plasma beta parameter, as both differ only by a factor of order unity in a stratified disk in vertical hydrostatic equilibrium.
}

gradients may also be present in disks as a result of the dynamics, and it is of some interest to explore values of $\epsilon$ of order unity as well.

\section{Analytic results}

It is of some interest to look into the linear behavior of the instability first, as it does provide some insight into the turbulent problem that we discuss in the next section. Indeed, there is evidence that the channel mode (both a linear and nonlinear solution to the incompressible equations) plays some role in the overall transport properties of the MRI-driven turbulent states.

For this reason, we want to test the turbulent resistivity hypothesis (Eqs. (10) and (11)) in the linear regime. This is done assuming the fluctuations $v$ and $b$ are infinitely small compared to the averaged fields. We then calculate $v$ and $b$ by a classical linear analysis and deduce the EMF $\mathcal{E}$ which is a quadratic quantity in this $\operatorname{limit}^{3}$. The same procedure can be used for Maxwell and Reynolds stresses, allowing one to compute "effective" transport coefficients in the linear regime.

To make comparisons easier, we investigate one of the configurations which is simulated in the next section of this paper, namely we impose a vertical mean field of the form

$B_{T}(y)=B_{0}\left[1+\epsilon \cos \left(k_{0} y\right)\right]$

where $k_{0}=2 \pi / L(=2 \pi$ in our choice of units) and $\epsilon$ measures the relative amplitude of the sinusoidal component of the mean field, and is to be taken as a small expansion parameter later on in some of the analytic expressions. Other configurations would lead to a similar qualitative behavior, which is the point of interest here.

Assuming that the development of the instability is fast compared to the viscous and resistive time-scales, we neglect the corresponding terms in Eqs. (5) and (6) in this linear analysis. Under this approximation, the system Eq. (5) to (7) admits a simple equilibrium with constant pressure and varying equilibrium azimuthal velocity ${ }^{4}$ profile to balance the magnetic pressure gradient:

$V(y)=S y-\frac{1}{4 \Omega_{0}} \frac{\mathrm{d} B_{\mathrm{T}}^{2}}{\mathrm{~d} y}$.

This leads us to introduce the total shear $S_{\mathrm{T}}$ :

$S_{\mathrm{T}}=S-\frac{1}{4 \Omega_{0}} \frac{\mathrm{d}^{2} B_{\mathrm{T}}^{2}}{\mathrm{~d} y^{2}}$.

It turns out that only channel-like mode present correlations of the EMF that behave as expected under the resistivity hypothesis, so we specialize from the onset to that type of modes. First order linear deviations from the mean stationary solution described above are referred to as $\boldsymbol{U}_{1}, \boldsymbol{B}_{1}, P_{1}$, and one considers only axisymmetric perturbations of the form

$\boldsymbol{X}_{1}(y, z, t)=\boldsymbol{x}(y) \exp (\mathrm{i} \omega t-\mathrm{i} k z)$,

\footnotetext{
${ }^{3}$ For this reason, this approach is also called "quasi-linear".

4 An actual disk is indeed expected to react to the presence of a superimposed magnetic pressure gradient at some initial time by first adjusting its velocity profile instead of its gas pressure profile. Nevertheless, we have also explored the converse situation, where the magnetic pressure variation is balanced by a variation of the gas pressure, without modification of the Keplerian profile (a behavior expected in an incompressible fluid). This change of equilibrium has little effect on the results reported in this section.
} 
where $\boldsymbol{X}, \boldsymbol{x}$ refers to the velocity, magnetic, and gaz pressure fields.

It is useful to distinguish the poloidal and toroidal components of the velocity and magnetic fields:

$\boldsymbol{v}=\boldsymbol{v}_{\perp}+v_{x} \boldsymbol{e}_{x}$,

$\boldsymbol{b}=\boldsymbol{b}_{\perp}+b_{x} \boldsymbol{e}_{x}$.

Because, of the assumed axisymmetry, on can introduce potential fields $\psi$ and $\varphi$ for these poloidal components such that

$\boldsymbol{v}_{\perp}=\mathrm{i} k \psi \boldsymbol{e}_{y}+\frac{\mathrm{d} \psi}{\mathrm{d} y} \boldsymbol{e}_{z}$,

$\boldsymbol{b}_{\perp}=\mathrm{i} k \varphi \boldsymbol{e}_{y}+\frac{\mathrm{d} \varphi}{\mathrm{d} y} \boldsymbol{e}_{z}$.

All variables can be expressed in terms of $\psi$, which leads to a second order ODE for $\psi$. One obtains:

$$
\begin{aligned}
\left(\omega^{2}-\right. & \left.V_{\mathrm{A}}^{2} k_{z}^{2}\right) \psi^{\prime \prime}-2 V_{\mathrm{b}}^{2} k_{z}^{3} \psi^{\prime} \\
+ & {\left[\left(\omega^{2}-V_{\mathrm{A}}^{2} k_{z}^{2}\right)-\frac{\kappa_{\mathrm{T}}^{2} \omega^{2}+2 \Omega_{0} S_{\mathrm{T}} V_{\mathrm{A}}^{2} k_{z}^{2}}{\omega^{2}-V_{\mathrm{A}}^{2} k_{z}^{2}}\right] k_{z}^{2} \psi=0, }
\end{aligned}
$$

where $V_{\mathrm{A}}(y)$ and $V_{\mathrm{b}}(y)$ are local Alfvén speed type quantities, and $\kappa_{\mathrm{T}}(y)$ is a local epicyclic frequency, defined by

$$
\begin{aligned}
V_{\mathrm{A}}^{2} & =B_{\mathrm{T}}^{2}, \\
V_{\mathrm{b}}^{2} & =\frac{B_{\mathrm{T}} B_{t}^{\prime}}{k_{z}}, \\
\kappa_{\mathrm{T}}^{2} & =2 \omega_{0}\left(2 \Omega_{0}-S_{\mathrm{T}}\right) .
\end{aligned}
$$

The magnetic field $\boldsymbol{b}$ and the azimuthal velocity $v_{x}$ can be expressed in terms of $\psi$ as follows:

$b_{x}=-\frac{k_{z} B_{\mathrm{T}}}{\omega} v_{x}-\frac{k_{z}^{2} S_{\mathrm{T}} B_{\mathrm{T}}}{\omega^{2}} \psi$,

$b_{y}=-\mathrm{i} \frac{k_{z}^{2} B_{\mathrm{T}}}{\omega} \psi$,

$b_{z}=-\frac{k_{z} B_{\mathrm{T}}}{\omega} \psi^{\prime}-\frac{k_{z} B_{\mathrm{T}}^{\prime}}{\omega} \psi$,

$v_{x}=-k_{z} \psi \frac{\omega^{2}\left(S_{\mathrm{T}}-2 \Omega_{0}\right)-S_{\mathrm{T}} V_{\mathrm{A}}^{2} k_{z}^{2}}{\omega\left(\omega^{2}-V_{\mathrm{A}}^{2} k_{z}^{2}\right)}$.

We have solved Eq. (22) numerically with periodic radial boundary conditions ${ }^{5}$. Analytic solutions can be found in terms of series expansion in $\epsilon$ in the form $\psi=\psi_{0}+\psi_{1}+\psi_{2} \ldots$ and $\omega^{2}=\omega_{0}^{2}+\omega_{1}^{2}+\omega_{2}^{2} \ldots$; the first order solution is rather straightforward to derive, but the explicit form of this solution is not particularly illuminating and will not be given in detail, except for some relevant features that will be mentioned whenever needed.

Solutions in the $\epsilon=0$ limit are the usual MRI modes, i.e. simple sinusoidal modes $\psi_{0} \propto \exp \left( \pm \mathrm{i} k_{y} y\right)$. Solutions for arbitrary $\epsilon$ generalize these modes. It turns out that only the nodeless mode $\left(k_{y}=0\right)$ produces a mean emf that correlates with the imposed current ${ }^{6}$, and in the following, only these modes are studied.

5 No boundary condition is implied vertically so that the modes are not discretized in this direction. In this section, the unit of length is set to $L_{y}$, but this has no incidence on the results or on the rest of the paper.

${ }_{6}$ The reason for this can be understood by looking at the equation for the first order correction $\psi_{1}$, which shows that the correct radial behavior for coupling occurs only in this case.

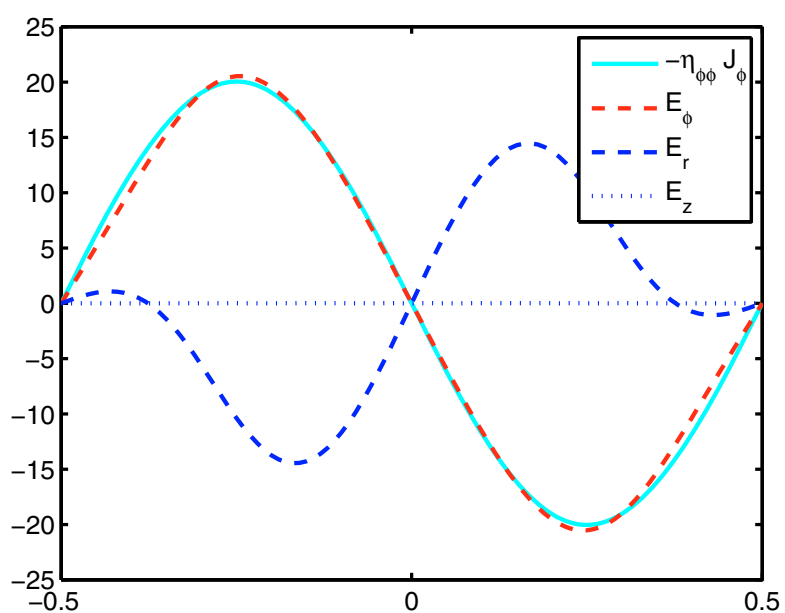

Fig. 1. Mean emfs and best fit with the current for $\beta=100$ and $\epsilon=0.3$ for the fastest growing channel mode with an imposed vertical mean field and radially sinusoidally varying imposed azimuthal current. The relevant emf component $E_{\phi}$ is seen to behave as a resistive term. This component $E_{\phi}$ measures the diffusion of the vertical field in the radial direction. The amplitudes are arbitrary.

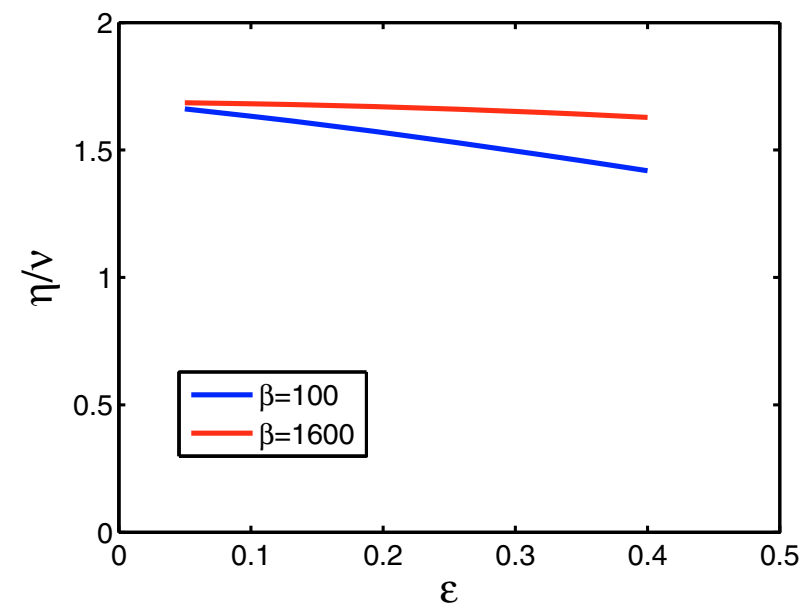

Fig. 2. Ratio of the mean emf $E_{\phi}$ and mean Reynolds stress tensor $(r, \phi)$ component due to the most unstable channel mode.

For definiteness, we show results pertaining to the fastest growing mode, for $\beta=100$ and $\epsilon=0.3$. Similar results are obtained for other sets of parameters, and for other purely vertical modes.

Following the quasi-linear analysis procedure, we define the quadratic emf as:

$\langle\mathcal{E}(y)\rangle=\frac{1}{2} \mathfrak{R}\left[\boldsymbol{v} \times \boldsymbol{b}^{\dagger}\right]$

where \langle\rangle denotes in this case a vertical average of the fluctuations, and the dagger stands for complex conjugation. As the channel mode is exponentially growing in time, the exponential prefactor has been left out (or equivalently, a time-dependent $\mathcal{E}$ would be required in the above equation). The various components of the emf due to the unstable mode, as well as the best fit of Eq. (11) with the imposed current are shown in Fig. 1.

It is of some interest to compare the efficiency of the "resistive" and "viscous" transport due to the instability, i.e., the value of $\eta_{\phi \phi}=e m f /$ current deduced here to the value of $v_{r \phi}=v=$ (Reynolds + Maxwell stress)/shear due to the same mode. This is shown in Fig. 2 for the same mode and for two different values 

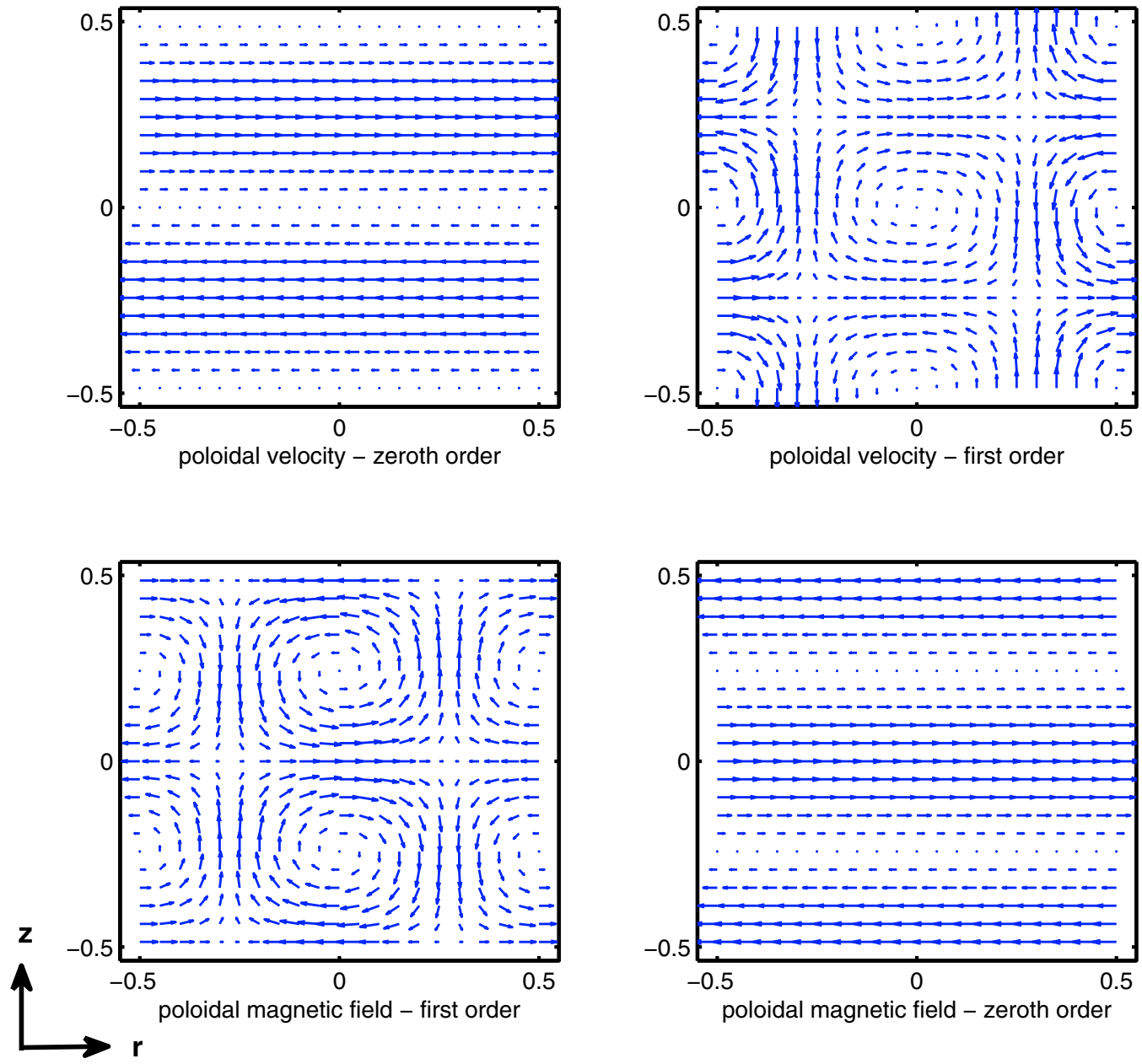

Fig. 3. Zeroth and first order components of the poloidal magnetic and velocity fields for the fastest growing channel mode $(\beta=100, \epsilon=0.3)$. The coupling of zeroth (resp. first) order component of the velocity field to the first (resp. zeroth) order component of the magnetic field gives rise to the transport of magnetic field by the channel mode.

of $\beta$ and a range of values of $\epsilon$. Note that this ratio is constant throughout the growth of the mode. The analytic result for this ratio in the limit of small $\epsilon$ is $\eta_{\phi \phi} / v_{r \phi}=27 / 16 \simeq 1.7$. Apparently, the relative efficiency of resistive and viscous transports due to the instability are of similar magnitude, more or less independently of the amplitude of the imposed field variation and mean field strength.

The comparable efficiency of the two transport effects due to the channel mode comes somewhat as a surprise. From a thermodynamical point of view, the instability sets in because of the imposed velocity shear and tries to restore a thermodynamical equilibrium by transporting momentum to reduce this shear. At first sight, there is no reason that it should also try to suppress a gradient in magnetic field with about the same efficiency.

However, if one considers the second-order correlation approximation used in mean field electrodynamic, one finds (e.g. Rädler \& Rheinhardt 2007):

$\beta_{i j} \sim \frac{1}{3}\left\langle\boldsymbol{v}^{2}\right\rangle \tau$

where $\tau$ is a typical correlation timescale of the small scale turbulence. This kind of result can be understood as a mixing length theory for magnetic fields involving velocities of the order of $v$ and a length on the order of $\tau v$. Although the secondorder correlation approximation is not entirely justified in the quasi-linear theory used here, at least some of the terms of the induction equation provide a mixing length behavior. This suggests that with a typical timescale on the order of the shear time, the dimensionless turbulent resistivity should be of the order of the turbulent transport (the turbulent transport being of the order of the turbulent kinetic energy). Pushing this argument one step further, we note that in general in numerical simulations, $\left\langle b_{x} b_{y}\right\rangle \sim 3\left\langle v_{x} v_{y}\right\rangle$, implying that one should observe $\beta_{i j} \sim \alpha / 4$. This is not consistent with the analytic result, but it agrees very well with the numerical results of the next section for a vertical field and a radial field variation; however it does not capture the origin of the significant anisotropy of the resistivity tensor observed in these simulations.

The analytic results as well as the preceding argument imply that the motion and magnetic field deviations due to the varying field should be coupled. An illustration of the efficiency of these couplings leading to this high resistive transport can be obtained from the analytic solution in the small $\epsilon$ limit. In this limit, the correlation arises from the correlation of zeroth and first order quantities in $\epsilon,\langle\boldsymbol{v} \times \boldsymbol{b}\rangle=\left\langle\boldsymbol{v}_{o} \times \boldsymbol{b}_{1}+\boldsymbol{v}_{1} \times \boldsymbol{b}_{0}\right\rangle$. Figure 3 shows the four involved poloidal fields for the most unstable channel mode $(\beta=100, \epsilon=0.3)$. Direct inspection indeed shows that the relevant fields do exhibit a significant level of correlation.

Having gained this understanding of the transport due to the linear modes of the instability, we now turn to the full nonlinear problem. 
Table 1. Main results from the $B_{z}(y)$ (vertical field with a radial dependency) case in the presence of a mean vertical field $B_{z}^{0}$.

\begin{tabular}{lccccc}
\hline \hline Model & $B_{z}^{0}$ & $\delta B^{0}$ & $\eta_{x x}$ & $\eta_{y x}$ & $\alpha$ \\
\hline ZZY1 & 0.1 & 0.01 & $3.6 \times 10^{-2}$ & $2.9 \times 10^{-2}$ & $1.5 \times 10^{-1}$ \\
ZZY2 & 0.1 & 0.02 & $3.7 \times 10^{-2}$ & $8.5 \times 10^{-2}$ & $1.5 \times 10^{-1}$ \\
ZZY3 & 0.1 & 0.03 & $3.6 \times 10^{-2}$ & $6.8 \times 10^{-2}$ & $1.5 \times 10^{-1}$ \\
ZZY4 & 0.1 & 0.04 & $2.4 \times 10^{-2}$ & $5.6 \times 10^{-2}$ & $1.3 \times 10^{-1}$ \\
ZZY5 & 0.1 & 0.08 & $1.1 \times 10^{-2}$ & $1.1 \times 10^{-2}$ & $7.8 \times 10^{-2}$ \\
ZZY6 & 0.025 & 0.0025 & $4.1 \times 10^{-3}$ & $2.6 \times 10^{-2}$ & $3.7 \times 10^{-2}$ \\
ZZY7 & 0.025 & 0.005 & $1.2 \times 10^{-2}$ & $-1.2 \times 10^{-2}$ & $3.3 \times 10^{-2}$ \\
ZZY8 & 0.025 & 0.0075 & $7.0 \times 10^{-3}$ & $2.8 \times 10^{-2}$ & $3.7 \times 10^{-2}$ \\
ZZY9 & 0.025 & 0.01 & $5.1 \times 10^{-3}$ & $2.4 \times 10^{-2}$ & $3.5 \times 10^{-2}$ \\
ZZY10 & 0.025 & 0.015 & $8.4 \times 10^{-3}$ & $1.5 \times 10^{-2}$ & $3.9 \times 10^{-2}$ \\
ZZY11 & 0.025 & 0.02 & $8.5 \times 10^{-3}$ & $1.5 \times 10^{-2}$ & $3.3 \times 10^{-2}$ \\
\hline
\end{tabular}

\section{Numerical results}

We have simulated three different configurations, which combine a mean vertical and/or azimuthal field with a varying field component in the vertical or azimuthal direction, the direction of variation being either vertical or radial. This allows us to characterize a number of components of the turbulent resistivity tensor.

To classify our numerical results, we use the following naming convention: the first letter indicates the direction of the mean field, the second letter indicates the direction of the varying field and the third letter corresponds to the direction of the spatial dependency. We finally add a number to identify the simulations done in the same configuration but with different field intensity. For instance, run XZY7 have a mean field in the $x$ direction, a varying field in the $z$ direction with a spatial dependency in the $y$ direction.

The various configurations and results are presented in the corresponding subsections. They are then compared in the concluding subsection.

\subsection{Vertical field with radial dependency $B_{z}(y)$}

In this case, we force the following structure for the mean magnetic field:

$\left\langle B_{z}\right\rangle=B_{z}^{0}+\delta B^{0} \cos \left(\frac{2 \pi y}{L_{y}}\right)$.

The mean current is then written:

$\langle\boldsymbol{J}\rangle=\left\langle J_{x}\right\rangle \boldsymbol{e}_{\boldsymbol{x}}=-\delta B^{0} \frac{2 \pi}{L_{y}} \sin \left(\frac{2 \pi y}{L_{y}}\right) \boldsymbol{e}_{\boldsymbol{x}}$.

We are therefore looking for a correlation between $J_{x}$ and $\mathcal{E}$. We show in Fig. 4 an example of a simulation result with $B_{z}^{0}=0.1$ and $\delta B^{0}=0.04(\epsilon=0.4)$. The profiles are computed from an average in time and in the $(x, y)$ plane. From this figure, one gets a classical diagonal resitivity term of $\eta_{x x} \sim 5.6 \times 10^{-2}$. We also find an non diagonal term $\eta_{y x}=8 \times 10^{-2}$. We have repeated this kind of experience for various sets of parameters as summarized in Table 1.

The extraneous EMF component was already present at the linear level, as noted in the previous section, but with the opposite sign. We have not been able to understand the significance of this sign difference. In our simulations, this component plays no physical role (its rotational vanishes). This may not be a generic feature, in particular in more complex stratified settings. This component shows anyway that the turbulent state is anisotropic.
Table 2. Main results from the $B_{x}(y)$ (toroidal field with a radial dependency) case for several toroidal and vertical mean fields $B_{j}^{0}$.

\begin{tabular}{cccccc}
\hline \hline Model & $B_{z}^{0}$ & $B_{x}^{0}$ & $\delta B^{0}$ & $\eta_{z z}$ & $\alpha$ \\
\hline ZXY1 & 0.1 & 0.0 & 0.01 & $9.0 \times 10^{-2}$ & $1.4 \times 10^{-1}$ \\
ZXY2 & 0.1 & 0.0 & 0.02 & $8.6 \times 10^{-2}$ & $1.6 \times 10^{-1}$ \\
ZXY3 & 0.1 & 0.0 & 0.03 & $8.8 \times 10^{-2}$ & $1.5 \times 10^{-1}$ \\
ZXY4 & 0.1 & 0.0 & 0.04 & $8.8 \times 10^{-2}$ & $1.4 \times 10^{-1}$ \\
ZXY5 & 0.1 & 0.0 & 0.06 & $8.2 \times 10^{-2}$ & $1.6 \times 10^{-1}$ \\
ZXY6 & 0.1 & 0.0 & 0.08 & $8.0 \times 10^{-2}$ & $1.5 \times 10^{-1}$ \\
XXY1 & 0.0 & 0.1 & 0.01 & $7.4 \times 10^{-3}$ & $7.6 \times 10^{-3}$ \\
XXY2 & 0.0 & 0.1 & 0.02 & $1.6 \times 10^{-2}$ & $8.1 \times 10^{-3}$ \\
XXY3 & 0.0 & 0.1 & 0.03 & $1.4 \times 10^{-2}$ & $9.1 \times 10^{-3}$ \\
XXY4 & 0.0 & 0.1 & 0.04 & $1.4 \times 10^{-2}$ & $9.2 \times 10^{-3}$ \\
XXY5 & 0.0 & 0.1 & 0.06 & $1.3 \times 10^{-2}$ & $8.9 \times 10^{-3}$ \\
XXY6 & 0.0 & 0.1 & 0.08 & $2.0 \times 10^{-2}$ & $1.3 \times 10^{-2}$ \\
XXY7 & 0.0 & 0.1 & 0.10 & $2.1 \times 10^{-2}$ & $1.6 \times 10^{-3}$ \\
\hline
\end{tabular}

One may note a decrease of the turbulence efficiency in models ZZY4-ZZY5, which may be due to the fact that increasing $\delta B_{0}$ to high values leads to strong modification of the background field. Since $B_{z}^{0}=0.1$ corresponds to the maximum of the growth rate for the $k_{z}=2 \pi / L_{z}$ mode, increasing $\delta B^{0}$ always weakens the instability, which might explain these results. As expected, this effect is not observed in $B_{z}^{0}=0.025$ models, for which this explanation doesn't hold.

According to these results, and anticipating on the result of the last subsection where the correlations are compared in detail, we point out that, on average

$\eta_{x x} \sim 0.235 \alpha$.

The resulting ratio $\eta / v$ ( $v=\alpha$ with our choice of units) is substantially smaller than its linear counterpart, and consistent with the argument exposed in the previous section.

\subsection{Toroidal field with a radial dependency $B_{x}(y)$}

In this case, we impose the following field:

$\left\langle B_{z}\right\rangle=B_{z}^{0}$,

$\left\langle B_{x}\right\rangle=B_{x}^{0}+\delta B^{0} \cos \left(\frac{2 \pi y}{L_{y}}\right)$.

The mean current is then defined by:

$\langle\boldsymbol{J}\rangle=\left\langle J_{z}\right\rangle \boldsymbol{e}_{z}=\delta B^{0} \frac{2 \pi}{L_{t}} \sin \left(\frac{2 \pi y}{L_{y}}\right)$.

As in the previous case, we look for a correlation between $J_{z}$ and $\mathcal{E}$. We show in Fig. 5 an example of such a correlation for $B_{z}^{0}=0.1, B_{x}^{0}=0$ and $\delta B^{0}=0.02$, from which we get $\eta_{z z} \sim 0.08$. Note that the other components of the EMF are poorly correlated with the imposed large scale field, indicating that either no correlation exists or that the turbulent viscosity model does not capture its physics; as a consequence, we have not quantified off-diagonal components of the resistivity tensor. As previously, we reproduce this kind of run for a range of parameters (see Table 2).

Comparing turbulent transport coefficient turbulent resistivity with the $B_{z}(y)$ case, we note that no turbulence weakening occurs in the present case, consistently with the linear justification (the expected linear growth rate is not modified by a varying 

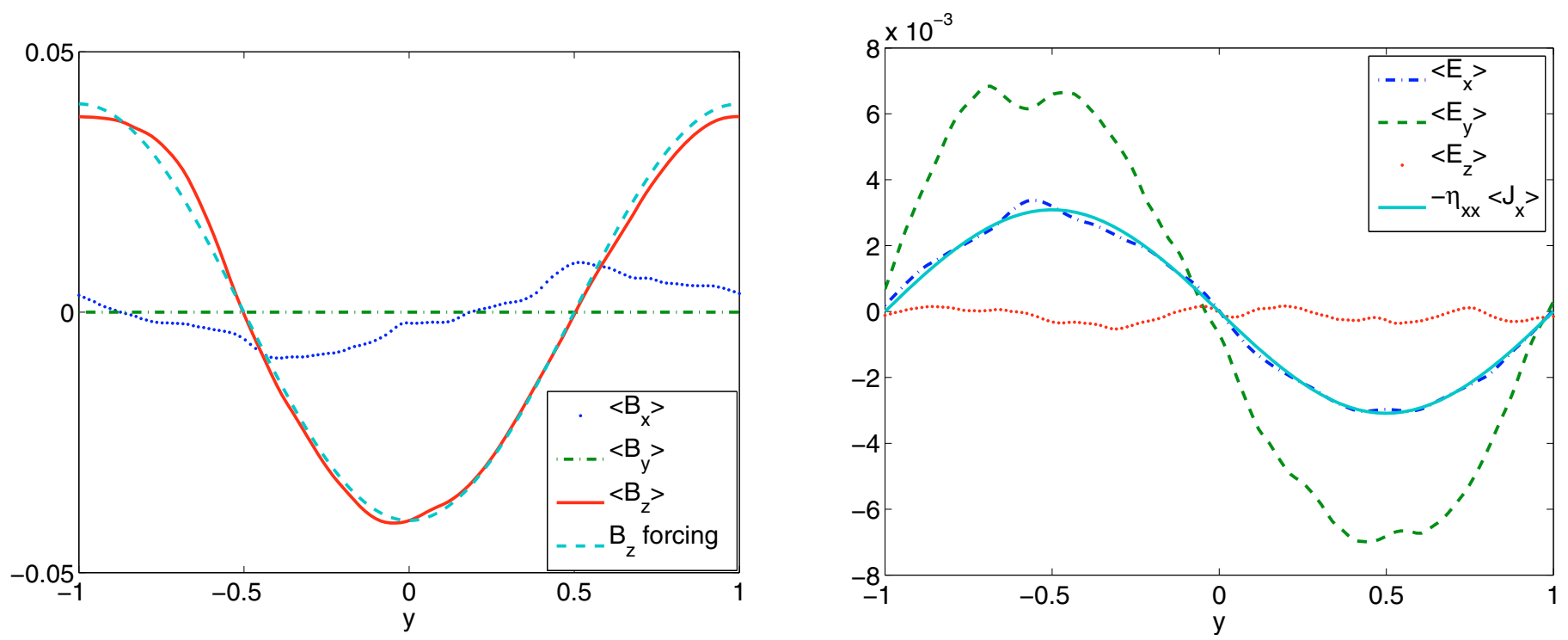

Fig. 4. Mean field (left $)$ and Emfs (right) from run ZZY4 $\left(B_{z}(y)\right.$ case) with a mean vertical field $B_{z}^{0}=0.1$ and $\delta B^{0}=0.04$. The right plot shows the best fit corresponding to the turbulent resistivity model. We measure in this case $\eta_{x x}=5.6 \times 10^{-2}$. Note that the mean field $B_{z}^{0}$ has been subtracted out.
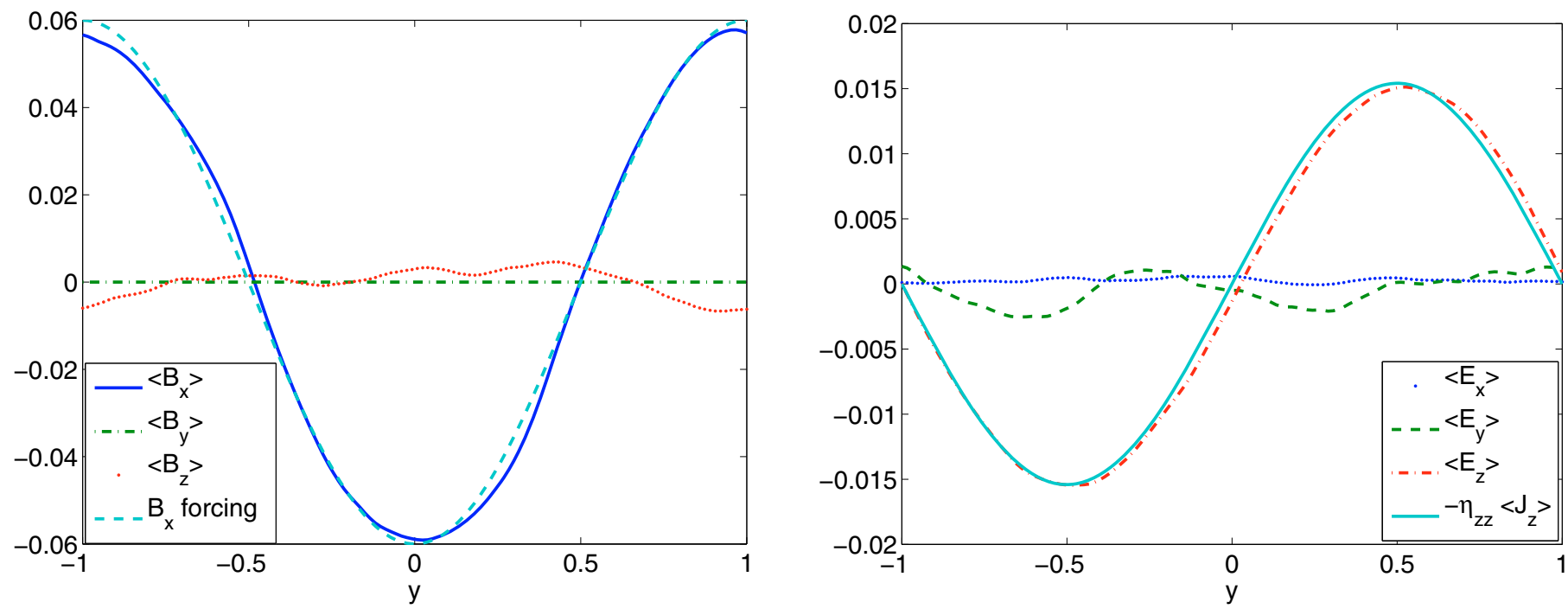

Fig. 5. Mean field (left $)$ and emfs (right) from run ZXY5 $\left(B_{x}(y)\right.$ case) with a mean vertical field $B_{z}^{0}=0.1$ and $\delta B_{0}=0.06$. The right plot shows the best fit corresponding to the turbulent resistivity model. We find in this case $\eta_{z z}=8.2 \times 10^{-2}$.

toroidal field). Although other experiments for larger values of $\beta$ would allow a better determination of the resistivity, we can still approximate

$\eta_{z z} \sim 0.57 \alpha$

with a mean vertical field and

$\eta_{z z} \sim 1.7 \alpha$

with a mean toroidal field.

\subsection{Toroidal field with a vertical dependency $B_{x}(z)$}

We define the mean field by:

$$
\begin{aligned}
& \left\langle B_{z}\right\rangle=B_{z}^{0}, \\
& \left\langle B_{x}\right\rangle=B_{x}^{0}+\delta B^{0} \cos \left(\frac{2 \pi z}{L_{z}}\right) .
\end{aligned}
$$

The mean current is then found to be:

$\boldsymbol{J}=J_{y} \boldsymbol{e}_{z}=\partial_{z} B_{x}$

$\langle\boldsymbol{J}\rangle=\left\langle J_{y}\right\rangle \boldsymbol{e}_{\boldsymbol{y}}=-\delta B^{0} \frac{2 \pi}{L_{z}} \sin \left(\frac{2 \pi z}{L_{z}}\right)$.

Looking for a correlation between $J_{y}$ and $\mathcal{E}$ is more problematic in this case. Indeed, if $B_{z}^{0} \neq 0$, the imposed $B_{x}(z)$ excites a channel flow solution, leading to the production of a large scale $B_{y}(z)$ (see Fig. 6). The existence of a persistent channel flow solution also leads to a double period $\mathcal{E}_{z}(z)$, as observed in the simulation.

These complications induce the existence of off-diagonal components of the resistivity tensor, which cannot be quantified with the simple method adopted in this paper. Nevertheless, in spite of these difficulties, the procedure used in the previous subsections yields reasonable results for the diagonal component, as can be seen in Fig. 6:

$\eta_{y y} \sim 0.08 \alpha$. 

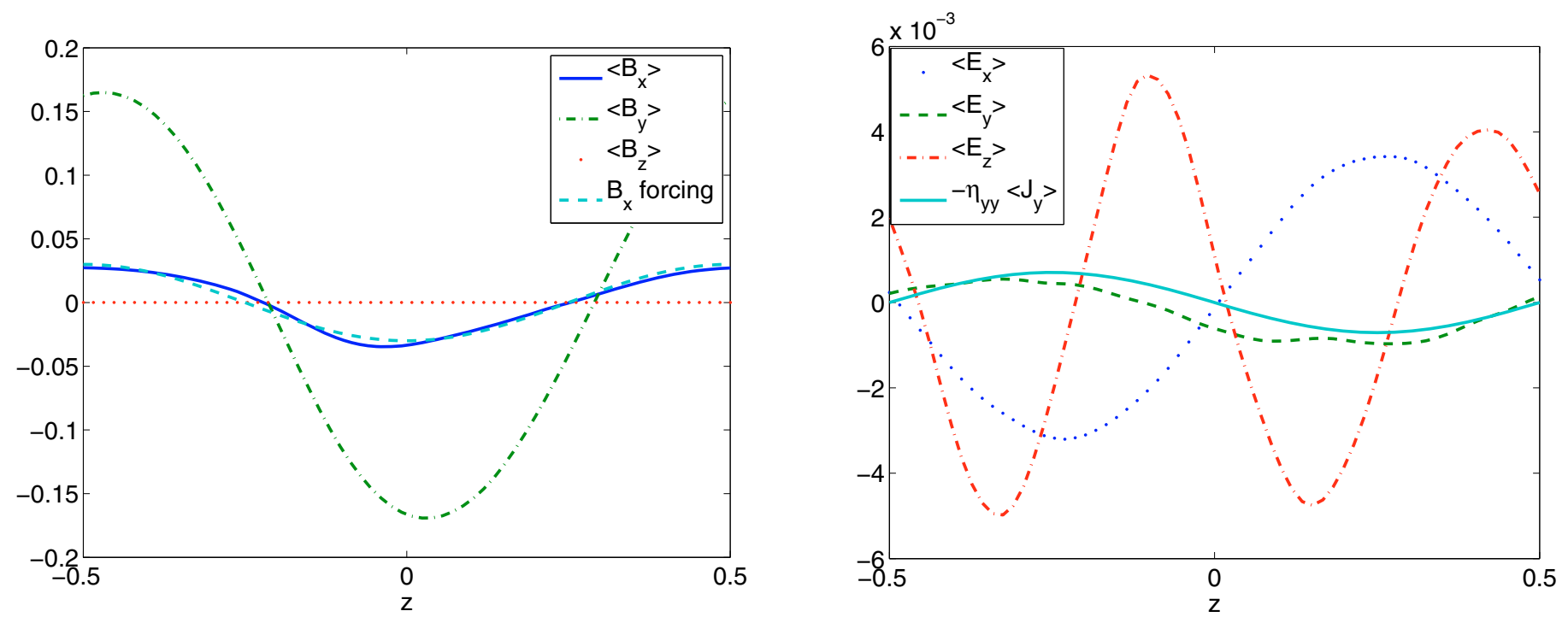

Fig. 6. Mean field (left) and emfs (right) for a case with $B_{z}^{0}=0.1$ and $\delta B^{0}=0.03$ and varying $B_{x}(z)$.

Table 3. Main results from the $B_{x}(z)$ (toroidal field with a vertical dependency) case with a mean vertical field $B_{z}^{0}$.

\begin{tabular}{ccccc}
\hline \hline Model & $B_{z}^{0}$ & $\delta B^{0}$ & $\eta_{y y}$ & $\alpha$ \\
\hline ZXZ1 & 0.1 & 0.01 & $4.8 \times 10^{-3}$ & $4.4 \times 10^{-2}$ \\
ZXZ2 & 0.1 & 0.02 & $2.5 \times 10^{-3}$ & $4.3 \times 10^{-2}$ \\
ZXZ3 & 0.1 & 0.03 & $3.7 \times 10^{-3}$ & $4.6 \times 10^{-2}$ \\
ZXZ4 & 0.1 & 0.04 & $4.8 \times 10^{-3}$ & $4.8 \times 10^{-2}$ \\
ZXZ5 & 0.1 & 0.06 & $6.8 \times 10^{-3}$ & $5.1 \times 10^{-2}$ \\
ZXZ6 & 0.1 & 0.08 & $3.2 \times 10^{-3}$ & $7.2 \times 10^{-2}$ \\
\hline
\end{tabular}

Table 4. Main results from the $B_{x}(z)$ (toroidal field with a vertical dependency) case for various toroidal $B_{x}^{0}$.

\begin{tabular}{cccccc}
\hline \hline Model & $B_{x}^{0}$ & $B_{z}^{0}$ & $\delta B^{0}$ & $\eta_{y y}$ & $\alpha$ \\
\hline XXZ1 & 0.1 & 0.0 & 0.01 & $5.1 \times 10^{-3}$ & $6.6 \times 10^{-3}$ \\
XXZ2 & 0.1 & 0.0 & 0.02 & $5.0 \times 10^{-3}$ & $7.8 \times 10^{-3}$ \\
XXZ3 & 0.1 & 0.0 & 0.03 & $5.6 \times 10^{-3}$ & $7.6 \times 10^{-3}$ \\
XXZ4 & 0.1 & 0.0 & 0.04 & $5.5 \times 10^{-3}$ & $8.1 \times 10^{-3}$ \\
XXZ5 & 0.1 & 0.0 & 0.06 & $5.1 \times 10^{-3}$ & $9.6 \times 10^{-3}$ \\
XXZ6 & 0.1 & 0.0 & 0.08 & $6.2 \times 10^{-3}$ & $1.2 \times 10^{-2}$ \\
XXZ7 & 0.1 & 0.0 & 0.1 & $6.0 \times 10^{-3}$ & $1.3 \times 10^{-2}$ \\
\hline
\end{tabular}

This value of $\eta$ is more uncertain than the other ones derived in this work, on the order of $30 \%$ to $50 \%$. The correlation with $\alpha$ is also not quite satisfied (see next subsection), but remains acceptable at the level of precision of determination of this quantity.

The value of $\eta_{y y}$ is more precisely derived when $B_{z}^{0}=0$ which prevent channel flow formation. We present in Fig. 7 an example of the profiles obtained from such a simulation and the resistivity values for several runs in Table 4.

Interestingly, $\eta_{y y}$ seems to be constant although $\alpha$ varies significantly, contrarily to previous cases. We can still approximate:

$\eta_{y y} \sim 0.72 \alpha$

We note however that this relation doesn't mean that the diffusive process is very strong in the vertical direction compared to that in the horizontal direction, as the turbulence intensity is weaker with a mean toroidal field than with a mean vertical field (compare for instance the values of $\alpha$ in Table 4 with models ZXY in Table 2). However, comparing only mean toroidal field simulations ( $\mathrm{X}^{* *}$ models), we can state that on average the $\eta_{y y}$ coefficient is significantly smaller than $\eta_{z z}$.

\subsection{Parameter and configuration dependence of the turbulent resistivity tensor}

It is of interest to compare the effect of the field configuration and magnitude on the various turbulent resistivity tensor components that we have characterized, most notably concerning the radial and vertical diffusion of vertical and azimuthal field components. To this effect, the various dependencies have been represented in Fig. 8.

The most significant trend is due to the direction of the mean field. As for turbulent viscous transport (but somewhat less dramatically), the turbulent resistive transport in the radial direction is substantially reduced when the mean field is azimuthal with respect to a vertical mean field, by nearly an order of magnitude; however, and somewhat surprisingly, no such trend is visible for the diffusion in the vertical direction (bottom left subplot). Our simulations with a mean toroidal field may suffer (as all others) from a lack of resolution of the relevant small scale modes, a point that has apparently never been adequately checked in the literature.

Another important trend, and the most critical one for the issue of jet driving from accretion disks, concerns the relative efficiency in the radial diffusion of vertical and azimuthal field components (top right subplot). The diffusion of the azimuthal field is more efficient by a factor of order 2 to 3 .

There is apparently some influence of the magnitude on the mean field on the efficiency of transport in the radial direction (top left subplot). This trend is the same as for the turbulent viscous transport, as shown on the bottom right subplot. The simplest explanation for this trend is that the transport is magnetically driven, whereas the usual adimensionalization of quantities (which we have more or less followed in our definitions) scales transport with the sound speed and the scale height: within factors of order unity, $v=\alpha c_{\mathrm{s}} H \sim \alpha \Omega H^{2}$ and similarly for the various $\eta$ components. With this scaling, the various simulations available in the literature imply that $\alpha \simeq 3 \beta^{-1 / 2}$. If a scaling with 

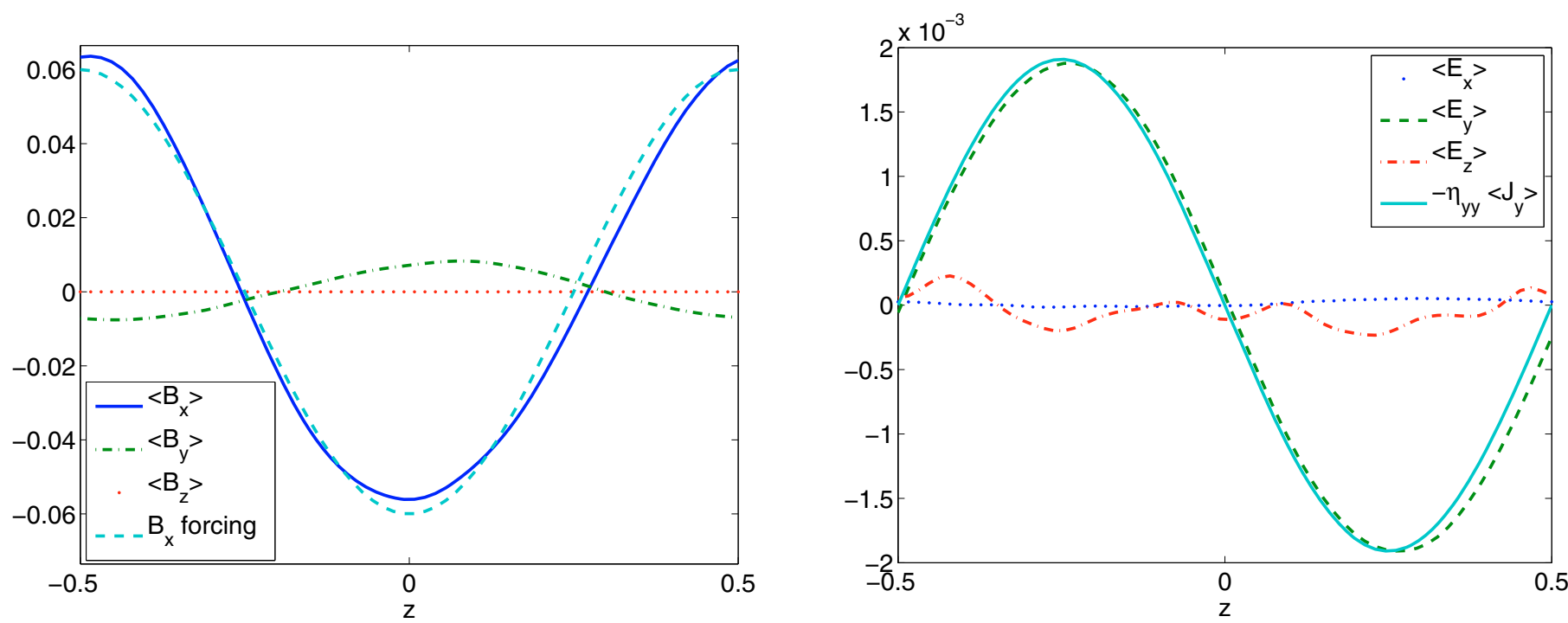

Fig. 7. Mean field $(l e f t)$ and emfs $(r i g h t)$ for a case with a mean toroidal flux $B_{x}^{0}=0.1, B_{z}^{0}=0, \delta B^{0}=0.06$ and varying $B_{x}(z)$. We deduce from this plot $\eta_{y y}=5.1 \times 10^{-3}$.
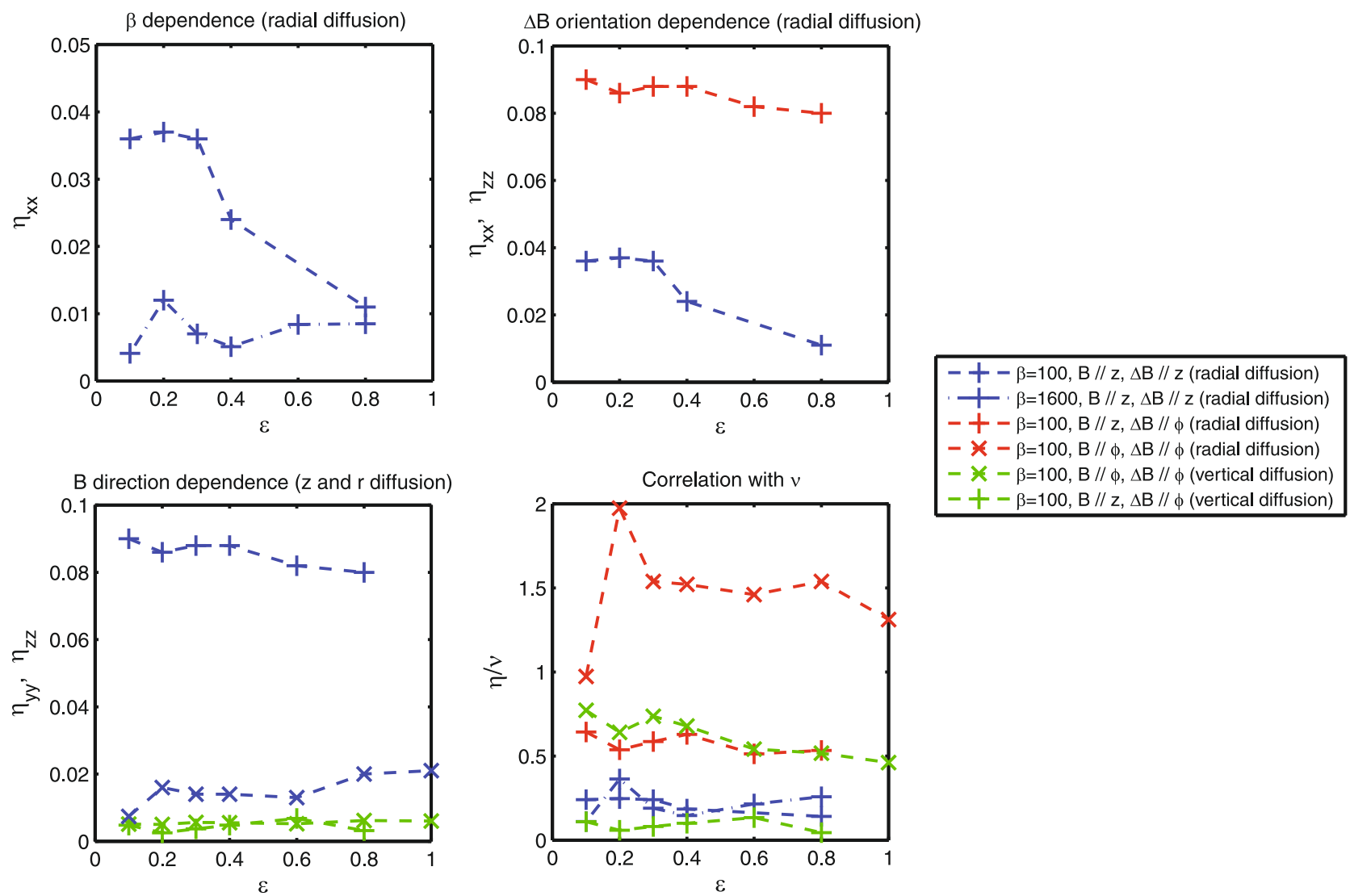

Fig. 8. Dependence of the turbulent diffusivity tensor components that we were able to measure on $\beta$ (relative field strength) and $\epsilon$ (relative amplitude of the varying field component), for various field configuration. The top left figure represents $\eta_{x x}$ for two different values of $\beta$. The bottom left one shows the role of the mean field orientation on $\eta_{z z}$. The top right compares $\eta_{x x}$ and $\eta_{z z}$. The bottom right figure shows the ratio of the relevant turbulent $\eta$ component over the turbulent viscosity. The symbols are the same as on the other subplots.

the Alfveén speed were chosen instead, i.e., $v=\alpha_{\mathrm{A}} v_{\mathrm{A}} H$, the characteristic dimensionless number $\alpha_{\mathrm{A}}$ is independent of $\beta$ (at least until $B_{0}$ is so weak that the zero mean field MRI dynamo process takes over). It has been noted in the first subsection that the decrease with $\epsilon$ for the largest value of $\beta$ is most likely a saturation process linked to the fact that this choice of $\beta$ is close to the marginal stability limit of the instability.
The last subplot (bottom right) indicates that $\beta$ (amplitude of the mean field) and $\epsilon$ (amplitude of the field variation) influence in much the same way the turbulent resistive and turbulent transport. To some extent, this is also true of the spatial orientation of the underlying quantities, and not only their magnitudes. Overall, the turbulent resistive transport has an efficiency which 
is smaller but comparable to the turbulent viscous one, within a factor of order 2 or 3.

\section{Discussion}

We have presented a systematic method to determine the turbulent resistivity associated with MRI turbulence in accretion disks. We have exemplified this method in the configuration radially and vertically varying vertical magnetic fields, using nonlinear spectral simulations of turbulence. We have also analyzed the resistive transport due to channel modes in the linear limit, in the presence of a radially varying vertical fields. Both the linear calculations and the fully nonlinear simulations show that the turbulent resistive transport is large, comparable to the turbulent viscous transport, albeit a factor of 2 to 4 smaller. This feature contradicts the heuristic model developed by Shu and coworkers (Shu et al. 2007) for turbulent resistive transport in YSOs accretion disks, thereby removing an obstacle to the launching of jets by accretion disks.

More precisely, we find that the turbulent resistivity $\eta^{\mathrm{T}}$ is largely correlated to the turbulent viscosity $v^{\mathrm{T}}$ over a wide range of variations of the dimensionless parameters of the problem (but we have not explored the dependency on the dissipation numbers, which needs an extensive study in itself). As a matter of fact, we may define a turbulent Prandtl number $P m^{T}=v^{\mathrm{T}} / \eta^{\mathrm{T}}$, which is found to be on the order of 2-5. Second, we find that the turbulent resistivity is an anisotropic tensor. In particular, the toroidal field $\left(B_{x}\right)$ diffuses about 3 times more rapidly than the poloidal field $\left(B_{z}\right)$, in the radial as well as the vertical directions. We also find that non diagonal terms of the turbulent resistivity tensor are non zero. As shown by Lesur \& Ogilvie (2008), such terms might play an important role for disk dynamos and large scale magnetic field generation.

It is often argued that a turbulent Prandtl number $\nu_{\mathrm{T}} / \eta_{\mathrm{T}}$ on the order of $R / H$ is required for turbulent disks to be able to launch jets (see van Ballegooijen in Belvedere 1989, and Lubow et al. 1994). In fact, because the accretion velocity in jet-driving disks is larger than in standard accretion disks, this requirement overestimates the necessary turbulent resistivity, which turns out to be comparable to the turbulent viscosity in self-consistent accretion-ejection models (see e.g. Casse \& Ferreira 2000; see also Rothstein \& Lovelace 2008 and Lovelace et al. 2009, for a simplified version of the same argument). This large turbulent Prandtl number argument is also often invoked to justify that an outer standard disk cannot transform into an inner jet-launching one in the accretion process, but the validity of this conclusion relies heavily on the assumed vertical structure of the models considered (in particular the magnetic structure of the corona), a point that has not been appropriately taken into account in the literature up to now.

On the basis of these results, it seems quite plausible that accretion disks have the ability to launch non stationary jets. Although the turbulent resistivity we find is somewhat too weak to allow for the existence of stationary accretion-ejection structure, the anisotropy is in the right range. Nevertheless, further work is required to get a complete characterization of the turbulent resistivity. In particular, the correlation with turbulent viscous transport needs to be more precisely studied, as well as the impact of the (molecular) Prandtl number, which is known to be strong on the momentum transport efficiency (Lesur \& Longaretti 2007).

While we were writing this paper, a similar study in shearing box with the ZEUS code has appeared on the astro-ph ArXiV by Guan and Gammie (Guan \& Gammie 2009), and some discussion of the connection between the two investigations is in order. In their paper, they impose of mean toroidal field, wait for turbulence to reach a stationary state. They superimpose then a sinusoidally varying field component whose decay rate is used to quantify the turbulent resistivity. Instead, we impose a constant sinusoidal component of the field and study the correlation between the resulting emf and the current in the insuing statistically stationary turbulent regime. Guan and Gammie have also made some resolution studies, which we have not performed, as previous experience with the dimensionless numbers used in this work has shown us that the dissipation scales are adequately resolved with our adopted resolution. Finally, Guan and Gammie have looked into the effect of the box aspect ratio, which was held fixed here. Conversely, our parameter study is somewhat more extensive than theirs for the type of configurations we have looked into. The majority of the runs performed by Guan and Gammie have been made for vertical sinusoidal component superimposed on the mean toroidal field, a configuration we have not investigated, so that these runs are not directly comparable to ours. Note however that using a mean vertical field instead of a mean toroidal one is more relevant to the question of flux diffusion for accretion-ejection models. Nevertheless, some of the runs of Guan \& Gammie (2009) have been performed with both the mean and sinusoidal component in the azimuthal direction, which are comparable to the ones presented here in Sect. 4.3. Their turbulent resistivity and viscosity values are systematically larger than ours, but note that their $\epsilon$ parameter in these runs is four times larger than the larger one we have used (and believe to be more relevant to disk physics). As there is a clear trend towards a significant increase of the turbulent resistivity and viscosity with increasing $\epsilon$ at the larger values of $\epsilon$ shown in Table 4, we conclude that the two results are reasonably compatible. More generally speaking, in order of magnitude, both studies are rather complementary and agree on the fact that the turbulent resistivity and viscosity are comparable, but runs in similar settings would be required to make a full comparison between the two methods.

Acknowledgements. The majority of the simulations presented in this paper were performed using the Darwin Supercomputer of the University of Cambridge High Performance Computing Service (http: //www . hpc . cam . ac . uk/), provided by Dell Inc. using Strategic Research Infrastructure Funding from the Higher Education Funding Council for England. G.L. acknowledges support by STFC. Other simulations have been performed on the SCCI cluster of the Observatoire de Grenoble.

We thank our referee, Jim Stone, for his detailed comments on this work.

\section{References}

Balbus, S. A. 2003, ARA\&A, 41, 555

Balbus, S. A., \& Hawley, J. F. 1991, ApJ, 376, 214

Belvedere, G., 1989, Astrophysics and Space Science Library, 156, Accretion disks and magnetic fields in astrophysics, Proceedings of the European Physical Society Study Conference, Noto, Italy, June 16-21, 1988

Bodo, G., Mignone, A., Cattaneo, F., Rossi, P., \& Ferrari, A. 2008, A\&A, 487, 1 Brandenburg, A., Rädler, K.-H., Rheinhardt, M., \& Käpylä, P. J. 2008, ApJ, 676, 740

Cabot, W. 1996, ApJ, 465, 874

Casse, F., \& Ferreira, J. 2000, A\&A, 353, 1115

Dubrulle, B., Dauchot, O., Daviaud, F., et al. 2005, Phys. Fluids, 17, 095103

Ferreira, J. 1997, A\&A, 319, 340

Fromang, S., Papaloizou, J., Lesur, G., \& Heinemann, T. 2007, A\&A, 476, 1123

Gammie, C. F. 1996, ApJ, 457, 355

Goodman, J., \& Xu, G. 1994, ApJ, 432, 213

Guan, X., \& Gammie, C. F. 2009, ApJ, 697, 1901 
Hawley, J. F. 2000, ApJ, 528, 462

Hawley, J. F., Gammie, C. F., \& Balbus, S. A. 1995, ApJ, 440, 742

Heinemann, T., \& Papaloizou, J. C. B. 2008a, MNRAS, 397, 52

Heinemann, T., \& Papaloizou, J. C. B. 2008b, MNRAS, 397, 64

Ji, H., Burin, M., Schartman, E., \& Goodman, J. 2006, Nature, 444, 343

Johnson, B. M., \& Gammie, C. F. 2006, ApJ, 636, 63

Klahr, H. H., \& Bodenheimer, P. 2003, ApJ, 582, 869

Lesur, G., \& Longaretti, P.-Y. 2005, A\&A, 444, 25

Lesur, G., \& Longaretti, P.-Y. 2007, MNRAS, 378, 1471

Lesur, G., \& Ogilvie, G. I. 2008, A\&A, 488, 451

Lovelace, R. V. E., Bisnovatyi-Kogan, G. S., \& Rothstein, D. M. 2009, Nonlinear Processes in Geophysics, 16, 77

Lubow, S. H., Papaloizou, J. C. B., \& Pringle, J. E. 1994, MNRAS, 267, 235

Petersen, M. R., Stewart, G. R., \& Julien, K. 2007, ApJ, 658, 1252
Peyret, R. 2002, Spectral Methods for Incompressible Viscous Flow (Springer) Rädler, K.-H., \& Rheinhardt, M. 2007, Geophysical and Astrophysical Fluid Dynamics, 101, 117

Regev, O., \& Umurhan, O. M. 2008, A\&A, 481, 21

Richard, D., \& Zahn, J. 1999, A\&A, 347, 734

Rothstein, D. M., \& Lovelace, R. V. E. 2008, ApJ, 677, 1221

Schrinner, M., Rädler, K.-H., Schmitt, D., Rheinhardt, M., \& Christensen, U. 2005, Astron. Nachr., 326, 245

Shakura, N. I., \& Sunyaev, R. A. 1973, A\&A, 24, 337

Shu, F. H., Galli, D., Lizano, S., \& Cai, M. J. 2007, in IAU Symp. 243, ed. J. Bouvier, \& I. Appenzeller, 249

Stone, J. M., \& Balbus, S. A. 1996, ApJ, 464, 364

Stone, J. M., Hawley, J. F., Gammie, C. F., \& Balbus, S. A. 1996, ApJ, 463, 656

Umurhan, O. M., \& Regev, O. 2004, A\&A, 427, 855 